\title{
Design of Few-Mode Fibers With Up to 12 Modes and Low Differential Mode Delay
}

\author{
Filipe Ferreira' ${ }^{1,2}$, Student Member, IEEE, Daniel Fonseca ${ }^{2,3}$, and Henrique Silva ${ }^{1}$, Member, IEEE \\ ${ }^{1}$ Instituto de Telecomunicações, Department of Electrical and Computer Engineering, University of Coimbra, \\ 3030-290 Coimbra, Portugal (filipe.ferreira@ co.it.pt) \\ ${ }^{2}$ Coriant Portugal, Unipessoal Lda., 2720-093 Amadora, Portugal \\ ${ }^{3}$ Instituto de Telecomunicações, Technical University of Lisbon, 1049-001 Lisboa, Portugal
}

\begin{abstract}
In this paper, we investigate the design of few-mode fibers (FMFs) guiding 4 to 12 non-degenerate linearly polarized (LP) modes with low differential mode delay (DMD) over the C-band, suitable for long-haul transmission. The refractive index profile considered is composed by a graded-core with a cladding trench (GCCT). The optimization of the profile parameters aims the lowest possible DMD and macro-bend losses (MBL) lower than the ITU-T standard recommendation. The optimization results show that the optimum DMD and the MBL scale with the number of modes. Additionally, it is shown that the refractive-index relative difference at the core center is one of the most preponderant parameters, allowing to reduce the DMD at the expense of increasing MBL. Finally, the optimum DMD obtained for 12 LP modes is lower than $3 \mathrm{ps} / \mathrm{km}$.
\end{abstract}

Keywords: Few-mode fibers, differential mode delay, refractive-index profile.

\section{INTRODUCTION}

Mode-division multiplexing (MDM) over few-mode fibers (FMFs) is emerging as an attractive solution for the impending capacity crunch of single-mode fibers (SMFs) [1] with potential cost, space, and energy savings [2]. However, FMFs require the usage of multiple-input multiple-output (MIMO) equalization to compensate for the combined effect of differential mode delay (DMD) and modal crosstalk (XT), which originates a channel impulse response (CIR) spread over time [3]. Consequently, the additional processing complexity partially erodes the benefit of deploying FMFs. It has been shown in [4] that, considering similar levels of complexity for nonlinearity mitigation in a standard SMF (SSMF), only FMF systems with 4 or more LP modes offer an actual capacity increase. Therefore, in this paper we investigate techniques to design FMFs with low DMD over the Cband from 4 to 12 LP modes.

In the literature, two different schemes have been proposed to limit the accumulation of DMD in FMFs with $x$ modes $(x \mathrm{M})$ : the usage of inherently low DMD FMFs (ILD-FMFs) [5], and the usage of DMD compensated FMFs (DC-FMFs) (FMFs with positive DMD followed by FMFs with negative DMD) [6]. The main target in this paper is a DMD lower than $12 \mathrm{ps} / \mathrm{km}$ over the C-band, since this is the DMD required for $2000 \mathrm{~km}$ of MDM transmission at $100 \mathrm{~Gb} / \mathrm{s}$ using an overhead of up to $10 \%$ [3]. The design of DC-FMFs with more than $2 \mathrm{M}$ requires the concatenation of a large number of FMFs with different DMDs, thereby imposing difficulties in the field deployment, compared to ILD-FMFs [7]. Therefore, in this paper we investigate only ILD-FMFs. The $x$ MILD-FMFs reported in the literature showing lower DMD over the C-band have been designed using a refractive index profile composed by graded-core with cladding trench (GCCT), for example: a 2M-ILD-FMF with $6 \mathrm{ps} / \mathrm{km}$ along $10 \mathrm{~km}$ [5], and a 4M-ILD-FMF with $135 \mathrm{ps} / \mathrm{km}$ along $7 \mathrm{~km} \mathrm{[6].} \mathrm{In} \mathrm{[9]} \mathrm{we} \mathrm{optimized} \mathrm{a} \mathrm{GCCT}$ profile for $4 \mathrm{M}$ and $6 \mathrm{M}$, obtaining DMD values of 5 and $10 \mathrm{ps} / \mathrm{km}$, respectively, over the C-band. Therefore, further improvement in the design of ILD-FMFs is required, in order to achieve cost-effective long-haul transmission systems.

In this work, the optimization of a GCCT profile is performed for $4 \mathrm{M}$ to $12 \mathrm{M}$, with the objective of obtaining a DMD lower than the $12 \mathrm{ps} / \mathrm{km}$ over the C-band requirement. The optimization of the GCCT profile presented in this paper reviews and extends the work that we presented in [8]-[10]. As the optimized DMD grows significantly with $x \mathrm{M}$ [9], in [10] we proposed to optimize the refractive index relative difference at the core center, $\Delta n_{c o}$, which was fixed in [8] and [9]. However, as $\Delta n_{c o}$ has a direct impact on the macro-bend losses (MBL) [11], we take into account such impact on the optimization function. The paper is organized as follows. Section 2 describes the profile considered and provides a theoretically explanation of the impact of $\Delta n_{c o}$ on DMD and MBL. Section 3 presents the optimization function algorithm. Section 4 presents the optimization results. Section 5 summarizes the main conclusions of this paper.

\section{Refractive-index Profile Description and Analysis}

In this paper, we follow the mathematical description of the GCCT profile presented in [8]. The core is characterized by the $\Delta n_{c o}$, the power-law exponent $\alpha$, and the core radius $w_{1}$. The trench dimensioning is characterized by the radial distance to the end of the core, $w_{2}$, the trench width $w_{3}$, and the relative refractive index difference at the trench $\Delta n_{t r}$. During the optimization, the guided modes (LP $\left.\mu v\right)$ and their characteristics are calculated solving the Maxwell equations numerically using the method described in [12]. The LP $\mu \nu$ mode 
characteristics calculated are the effective index $\bar{n}^{\mathrm{LP} \mu v}$, the effective group index $\bar{n}_{g}{ }^{\mathrm{LP} \mu v}$, the DMD and the MBL. The DMD of the LP $\mu \nu$ mode is measured relatively to the LP01 mode and is given by $D M D_{L P \mu v}(\lambda)=\left[\bar{n}_{g}{ }^{\mathrm{LP} \mu v}(\lambda)-\bar{n}_{g}{ }^{\mathrm{LP} 01}(\lambda)\right] / c$, where $\lambda$ is the wavelength and $c$ is the light velocity in vacuum. The MBL are calculated according to [11]. The dispersion properties of the doped silica have been modeled using the Sellmeier coefficients used in [8].

When designing a graded core fiber with a given number of modes, one must first choose the normalized frequency $(V)$ value. $V$ is given by $V=2 \pi w_{1} / \lambda \cdot\left[n_{c o}{ }^{2}-n_{c l}{ }^{2}\right]^{1 / 2}$ where $n_{c o}$ is the refractive-index value at the core center and $n_{c l}$ is the refractive-index value at the cladding. For each $x \mathrm{M}$ fiber, we choose the highest possible $V$ value that guarantees the guidance of the first $x$-modes while cutting off the next higher-order modes [10], considering a GCCT profile with $\alpha=2.3$ and $\Delta n_{t r}=0$. As a result, for $4 \mathrm{M}, 6 \mathrm{M}, 9 \mathrm{M}$ and $12 \mathrm{M}$, the $V$ values are chosen to be $7.25,9.00,11.15$ and 12.95 , respectively. As a consequence, the $x$-modes have the highest possible $\bar{n}^{-\mathrm{LP} \mu v}$ values and are thus more strongly guided. Given $V$ and $\Delta n_{c o}$, the $w_{1}$ value is obtained considering the lowest $\lambda$ of the C-band $(1530 \mathrm{~nm})$. Along this paper, references to a $\Delta n_{c o}$ change imply a $w_{1}$ change such that $V$ remains constant.

In the following, the impact of $\Delta n_{c o}$ value on the DMD of a GCCT profile is explained theoretically. The $\Delta n_{c o}$ value limits the maximum difference possible between the effective indexes $\bar{n}^{\mathrm{LP} \mu v}$, since $n_{c l}<\bar{n}^{\mathrm{LP} \mu v}<n_{c o}$. Consequently, the $\Delta n_{c o}$ value also limits the maximum difference possible between effective group indexes $\bar{n}_{g}{ }^{\mathrm{LP} \mu \nu}$, since $\bar{n}_{g}{ }^{\mathrm{LP} \mu v}=\bar{n}^{-\mathrm{LP} \mu v}+\lambda \cdot\left[d \bar{n}^{\mathrm{LP} \mu v} / d \lambda\right]$. Noting that $D M D_{L P \mu \nu}(\lambda)=\left[\bar{n}_{g}{ }^{\mathrm{LP} \mu v}(\lambda)-\bar{n}_{g}{ }^{\mathrm{LP} 01}(\lambda)\right] / c$, it can be concluded that the reduction of $\Delta n_{c o}$ has the potential to further reduce the DMD values obtained in [9]. The drawback of the utilization of a low $\Delta n_{c o}$ is related to MBL. According to [11], the power loss at bends increases with decreasing $\Delta n_{c o}$ for a certain curvature radius and, as a consequence, low DMD and low MBL are opposite requirements. The trade-off between DMD and MBL on the optimization of $\Delta n_{c o}$ is analyzed in Section 4.

\section{Optimization Function and Algorithm}

The optimization parameters can be gathered in a parameter vector $(p v): p v=\left[\alpha, \Delta n_{c o}, w_{2}, w_{3}, \Delta n_{t r}\right]$. The optimization function takes into account two figures: one related to DMD and another related to MBL. The DMD related figure is the maximum DMD among the guided modes and over the defined wavelength range $(\max D M D)$, given by:

$$
\max D M D(p v)=\max _{\lambda}\left(\max _{\mu v}\left|D M D_{L P \mu v}(\lambda, p v)\right|\right)
$$

The MBL related figure is the curvature radius $\left(R_{c}\right)$ for 100 turns and $\mathrm{MBL}=0.1 \mathrm{~dB}$ at $1625 \mathrm{~nm}$. For a given $x \mathrm{M}$ fiber, the $R_{c}$ of each mode is calculated and the highest value is considered. According to the ITU-T recommendation in [13], $R_{c}$ must be lower than or equal to $30 \mathrm{~mm}$. The optimization function $(O F)$ is given by (2) and the respective constraints are given by (3)-(8).

$$
\begin{aligned}
& O F(p v)=\max D M D(p v) \cdot\left\{1+\varepsilon \cdot\left[\beta \cdot \frac{\left(R_{c}-30\right)}{30}\right]\right\} \\
& \beta=\left\{\begin{array}{l}
0, \text { for } R_{c} \leq 30 \\
1, \text { for } R_{c}>30
\end{array}\right. \\
& \text { with } R_{c} \text { in millimeter units. }
\end{aligned}
$$

$$
\begin{gathered}
\left(\Delta n_{c o}{ }^{-}=1 \cdot 10^{-3}\right) \leq \Delta n_{c o} \leq\left(\Delta n_{c o}{ }^{+}=5 \cdot 10^{-3}\right) \\
\left(\alpha^{-}=1.5\right) \leq \alpha \leq\left(\alpha^{+}=2.5\right) \\
\left(\Delta n_{t r}{ }^{-}=-5 \cdot 10^{-3}\right) \leq \Delta n_{t r} \leq\left(\Delta n_{t r}{ }^{+}=0\right) \\
\left(w_{2}{ }^{-}=0\right) \leq w_{2} \leq\left(w_{2}^{+}=w_{1} / 2\right) \\
\left(w_{3}^{-}=0\right) \leq w_{3} \leq\left(w_{3}^{+}=w_{1}\right) \\
\left(\lambda^{-}=1530 \mathrm{~nm}\right) \leq \lambda \leq\left(\lambda^{+}=1565 \mathrm{~nm}\right)
\end{gathered}
$$

In (2), the $\varepsilon$ factor can be 0 or 1 in order to consider or ignore the $R_{c} \leq 30 \mathrm{~mm}$ requirement. The $\beta \cdot\left(R_{c}-30\right) / 30$ factor in (2) introduces a penalizing factor for solutions with $R_{c}>30 \mathrm{~mm}$, since $\beta$ is equal to 0 for $R_{c} \leq 30 \mathrm{~mm}$ and equal to 1 for $R_{c}>30 \mathrm{~mm}$. Note that, for each different $p v$ tested, if the number of modes is not the desired one the $O F$ value is set to infinity. Regarding the constraints, $\Delta n_{c o}{ }^{-}$in (3) takes into account the difficulties of manufacturing fiber with $\Delta n_{c o}$ lower than $1 \cdot 10^{-3}$, whereas $\Delta n_{c o}{ }^{+}$is used as upper bound of $\Delta n_{c o}$ taking into account that $\max D M D$ increases with $\Delta n_{c o}$. (4)-(7) were defined in [8]. (8) binds $\lambda$ to the C-band.

The optimization algorithm is designed taking into account that maxDMD is a convex function of $\left(\alpha, \Delta n_{t r}\right)$ [10]. Therefore, the search for the pair $\left(\alpha, \Delta n_{t r}\right)$ that minimizes $\operatorname{maxDMD}$ for a given $\left(\Delta n_{c o}, w_{2}, w_{3}\right)$ point is done one dimension at a time using a golden section search (GSS). In order to find the full optimum $p v$ set, an exhaustive search (ES) is performed over $\left(\Delta n_{c o}, w_{2}, w_{3}\right)$. The GSS optimizes $\alpha$ and $\Delta n_{t r}$ with a termination 
tolerance on maxDMD of $0.001 \mathrm{ps} / \mathrm{km}$. The ES optimizes the $\Delta n_{c o}, w_{2}$ and $w_{3}$ with tolerances of $5 \cdot 10^{-4}, 0.25 \mu \mathrm{m}$ and $0.5 \mu \mathrm{m}$, respectively. Further reducing these tolerances by a factor of 2 changed $\max D M D$ negligibly.

\section{Optimization Results}

The optimization results are shown in this section. The $\max D M D$ and $R_{c}$ are required to be equal or lower than $12 \mathrm{ps} / \mathrm{km}$ and $30 \mathrm{~mm}$, respectively. Fig. 1 (a) and (b) show $\operatorname{maxDMD}$ and $R_{c}$ optimum values, respectively, as a function of the number of modes, obtained using $O F$ with $\varepsilon=0$ and $\varepsilon=1$. Fig. 1 (a) and (b) show, respectively, that $\max D M D$ and $R_{c}$ scale with the number of modes for a given $\Delta n_{c o}$ value and $\varepsilon=0$. Furthermore, Fig. 1 (a) and (b) show, respectively, that $\max D M D$ decreases and $R_{c}$ increases with $\Delta n_{c o}$ decreasing for a given number of modes and $\varepsilon=0$, in line with the explanation provided in Section 2. In particular, with $\varepsilon=0$, Fig. 1 (a) shows that the $\max D M D$ requirement is not satisfied for $x \mathrm{M}>6$ with $\Delta n_{c o}=5 \cdot 10^{-3}$, and Fig. 1 (b) shows that the $R_{c}$ requirement is not satisfied for any number of modes with $\Delta n_{c o} \leq 3 \cdot 10^{-3}$. Comparing the results shown in Fig. 1 obtained using $\varepsilon=0$ and $\varepsilon=1$, it can be concluded that the $R_{c}$ requirement can be satisfied from $4 \mathrm{M}$ to $12 \mathrm{M}$ with small maxDMD degradation (lower than $0.5 \mathrm{ps} / \mathrm{km}$ for $\Delta n_{c o}=1 \cdot 10^{-3}$ ). Therefore, Fig. 1 shows that the maxDMD and $R_{c}$ requirements are satisfied simultaneously for $1 \cdot 10^{-3} \leq \Delta n_{c o} \leq 4 \cdot 10^{-3}$ from $4 \mathrm{M}$ to $12 \mathrm{M}$. Moreover, it can be concluded that $\max D M D$ cannot be reduced to negligible levels $(\max D M D<0.1 \mathrm{ps} / \mathrm{km})$, as for $2 \mathrm{M}$ [8]. This limitation is explained noting that the field confinement effect of the trench affects each higher-order mode (LP02, LP21, LP12, LP31, ...) with different strength, since all have a considerable power concentration near the core boundary but different distributions [8]. Therefore, each mode has a different optimum trench dimensioning $\left(w_{2}, w_{3}, \Delta n_{t r}\right)$ and it is not possible to reduce the DMD of all modes to negligible values at the same time over the C-band. Fig. 2 shows the optimum trench dimensioning $\left(w_{2}, w_{3}, \Delta n_{t r}\right)$ as a function of the number of modes for $\Delta n_{c o} \cdot 10^{3}=\{1,2,3\}$. From Fig. 2 it can be seen that, for a given $\Delta n_{c o}$, when increasing the number of modes the

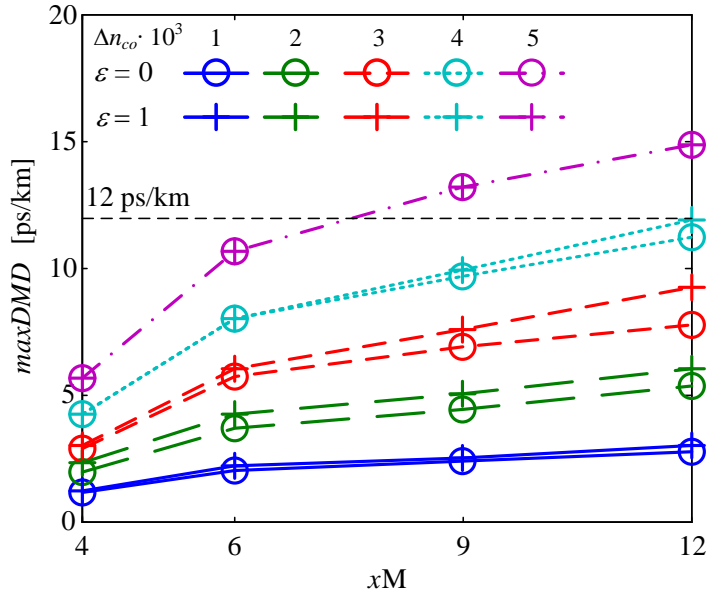

(a)

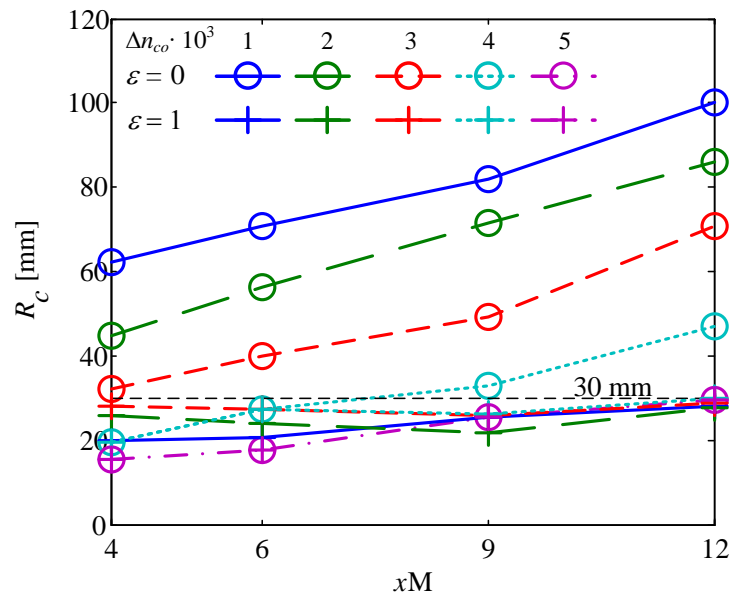

(b)

Figure 1. (a) maxDMD [ps/km] and (b) $R_{c}[\mathrm{~mm}]$ optimum values as a function of the number of modes for different $\Delta n_{\text {co }}$ values.

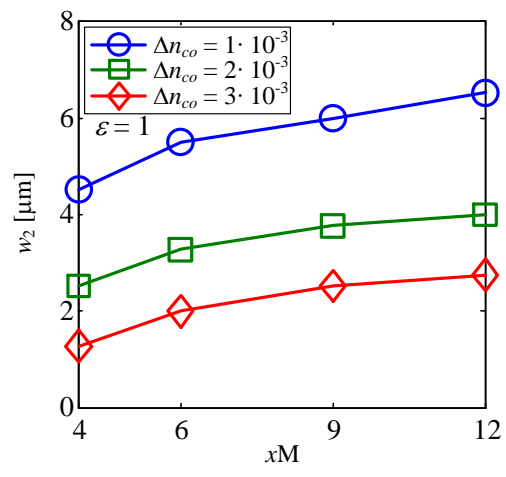

(a)

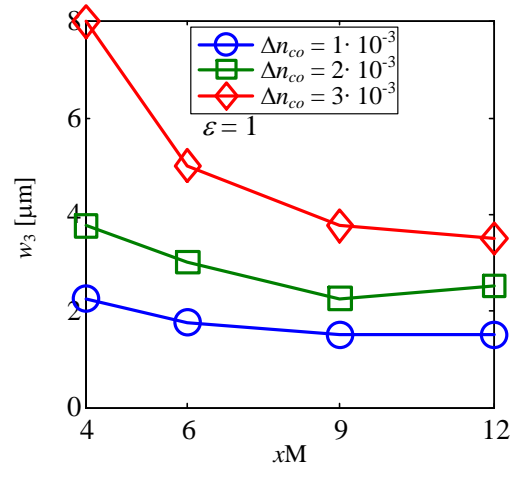

(b)

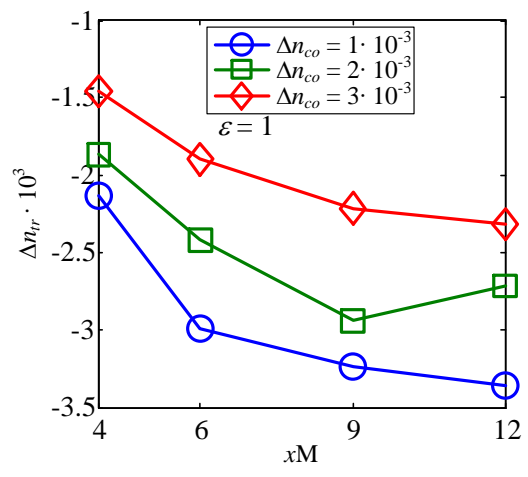

(c)

Figure 2. Optimum trench dimensioning $\left(w_{2}, w_{3}, \Delta n_{t r}\right)$ as a function of number of modes for $\Delta n_{c o} \cdot 10^{3}=\{1,2,3\}$. (a) $w_{2},(b) w_{3}$, and (c) $\Delta n_{t r}$. 
optimum trench gets farther away from the core ( $w_{2}$ increases), narrower ( $w_{3}$ decreases) and deeper $\left(\Delta n_{t r}\right.$ decreases). This means that the guidance of higher-order modes with different spatial distributions alters significantly all the optimum trench dimensioning parameters $\left(w_{2}, w_{3}, \Delta n_{t r}\right)$, as explained above. Additionally, Fig. 2 shows that, for a given number of modes, when increasing $\Delta n_{c o}$ the optimum trench gets closer to the core ( $w_{2}$ decreases), wider ( $w_{3}$ increases) and shallower $\left(\Delta n_{t r}\right.$ increases).

The remaining properties of the optimized FMFs for $\Delta n_{c o}=1 \cdot 10^{-3}$ and $\varepsilon=1$ have been computed for $1550 \mathrm{~nm}$ : the chromatic dispersion $(D)$, the chromatic dispersion slope $(S)$ and the nonlinear coefficient $(\gamma)$. The $D$ value is around $22 \mathrm{ps} / \mathrm{km} / \mathrm{nm}$ (from LP01 to the higher-order mode) for all the numbers of modes considered, only moderately higher than the dispersion of $\sim 17 \mathrm{ps} /(\mathrm{nm} \cdot \mathrm{km})$ characteristic of SSMF. The $S$ value is around 0.06 $\mathrm{ps} /\left(\mathrm{nm}^{2} \cdot \mathrm{km}\right)$ (from LP01 to the higher-order mode) for all the number of modes considered, lower than the value of $\sim 0.08 \mathrm{ps} /\left(\mathrm{nm}^{2} \cdot \mathrm{km}\right)$ typical of SSMF. The $\gamma$ value for the LP01 mode (the most restrictive one) goes from 0.22 for $2 \mathrm{M}$ to 0.09 for $12 \mathrm{M}$, significantly lower than the SSMF typical value of $1.3 \mathrm{~W}^{-1} / \mathrm{km}$, as expected due to the higher core radius.

As a main conclusion, the results presented in this section allow stating that optimizing $\Delta n_{c o}$ allowed to satisfy the requirements of $\max D M D \leq 12 \mathrm{ps} / \mathrm{km}$ and $R_{c} \leq 30 \mathrm{~mm}$, which were not achievable in [9].

\section{CONCLUSIONS}

In this work, the design of FMFs with low DMD over the C-band was investigated considering a GCCT profile. The profile parameters were optimized obtaining the lowest $\operatorname{maxDMD}$ achievable for $4 \mathrm{M}$ to $12 \mathrm{M}$, with $R_{c} \leq 30$ mm. The optimization results have shown that $\max D M D$ and $R_{c}$ scale with the number of modes. $\Delta n_{c o}$ was shown to be the most preponderant parameter of the GCCT profile, allowing reducing maxDMD at the expense of increasing $R_{c}$. The optimization results obtained for the lowest $\max D M D$ (ignoring the $R_{c}$ value) have shown that, for $\Delta n_{c o} \leq 3 \cdot 10^{-3}$, the $R_{c}$ requirement is not satisfied for any number of modes. On the other hand, optimizing simultaneously for low $\max D M D$ and low $R_{c}$, it was possible to satisfy the $\max D M D$ and $R_{c}$ requirements simultaneously for $1 \cdot 10^{-3} \leq \Delta n_{c o} \leq 4 \cdot 10^{-3}$ from $4 \mathrm{M}$ to $12 \mathrm{M}$, with a maxDMD penalty lower than $0.5 \mathrm{ps} / \mathrm{km}$. For $12 \mathrm{M}$ and $\Delta n_{c o}=1 \cdot 10^{-3}$, a $\max D M D$ lower than $3 \mathrm{ps} / \mathrm{km}$ was obtained.

\section{ACKNOWLEDGEMENTS}

This work was supported in part by Coriant Portugal Unipessoal Lda., in part by the Fundação para a Ciência e Tecnologia under Grant SFRH/BDE/51094/2010, and in part by the 7th Framework Programme of the European Community, under Grant 258033 (MODE-GAP).

\section{REFERENCES}

[1] A. Ellis, J. Zhao, and D. Cotter, “Approaching the Non-Linear Shannon Limit,” J. Lightw. Technol., vol. 28, no. 4, pp. 423-433, Feb. 15, 2010.

[2] P. Winzer, "Energy-Efficient Optical Transport Capacity Scaling Through Spatial Multiplexing," IEEE Photon. Technol. Lett., vol. 23, no. 13, pp. 851-853, July1, 2011.

[3] B. Inan, et al., "Equalizer complexity of mode division multiplexed coherent receivers," in Proc. OFC 2012, pp. 1-3, paper OW3D.4.

[4] A. Ellis, and N. Doran, "Are few-mode fibres a practical solution to the capacity crunch?," in Proc. ICTON 2013, pp. 1-4, paper Tu.C2.1.

[5] L. Gruner-Nielsen, et al., "Few mode transmission fiber with low DGD, low mode coupling and low loss," J. Lightw. Technol., vol. 30, no. 23, pp. 3693-3698, Dec. 2012.

[6] T. Mori, et al., "Low DMD Four LP Mode Transmission Fiber for Wide-band WDM-MIMO System," in Proc. OFC 2013, pp. 1-3, paper OTh3K.1.

[7] R. Ryf, et al., "32-bit/s/Hz Spectral Efficiency WDM Transmission over 177-km Few-Mode Fiber," in Proc. OFC 2013, pp. 1-3, paper PDP5A.1.

[8] F. Ferreira, D. Fonseca, and H. Silva, "Design of Few-Mode Fibers With Arbitrary and Flattened Differential Mode Delay," IEEE Photon. Technol. Lett., vol.25, no.5, pp.438-441, Mar. 1, 2013.

[9] F. Ferreira, D. Fonseca, and H. Silva, "On the Dependence of Differential Mode Delay in Few-Mode Fibers on the Number of Modes," in Proc. ICTON 2013, pp. 1-4, paper Tu.C2.3.

[10]F. Ferreira, D. Fonseca, and H. Silva, "Design of Few-Mode Fibers With $M$-modes and Low Differential Mode Delay,” J. Lightw. Technol., vol. 32, no. 3, pp. 353-360, Feb. 1, 2014.

[11] J. Sakai, and T. Kimura, "Bending loss of propagation modes in arbitrary-index profile optical fibers," Appl. Opt., vol. 17, no. 10, pp. 1499-1506, 1978.

[12] C. Yeh, and G. Lindgren, "Computing the propagation characteristics of radially stratified fibers: an efficient method," Appl. Opt., vol. 16, no. 2, pp. 483-493, 1977.

[13] Characteristics of a single-mode optical fibre cable, Standard ITU-T G.652B, Oct. 2010. 